\title{
Spectro-Polarimetrc Optical Systems For Imaging Plasma Internal Fields, Structures And Flows
}

\section{John Howard ${ }^{*} a$, Clive Michael $a$, Haitao Chen $a$, Romana Lester $a$, Alex Thorman $a$ and Jinil Chung $b$}

a Plasma Research Laboratory, Australian National University, Canberra 2601, AUSTRALIA

b National Fusion Research Institute, Daejeon, 305-333, Korea

E-mail: john.howardeanu.edu.au

\begin{abstract}
Spectro-polarimetric imaging systems have opened new and better ways to study the spatiotemporal behaviour of plasma properties including current distribution, pedestal fields and the velocity distribution function of radiating species from the plasma edge to the core. Using spatialheterodyne polarimetric techniques, these coherence-imaging (CI) systems have been deployed on many devices around the world, including for high resolution $(\sim 5 \mathrm{~mm}, \sim 5 \mathrm{~ms})$ motional Stark effect (MSE) imaging on KSTAR and ASDEX-Upgrade, and for passive Doppler spectroscopy of the plasma edge on DIII-D and MAST. In a partner paper submitted to Journal of Instrumentation, we describe the application of spatial-heterodyne polarization-interferometric techniques for snapshot imaging of the temporal coherence and Stokes parameters of plasma optical emission for Doppler spectroscopy and motional Stark effect polarimetry. In particular, we focus on the results of Doppler imaging of low-temperature argon discharges on the linear mirror device MAGPIE at the ANU including, for the first time, synchronous Doppler imaging of flow vortices (velocity resolution $\sim$ few $\mathrm{m} / \mathrm{s}$ ) associated with low frequency drift-type instabilities. We describe the diagnostic technologies, discuss the results and comparisons with simple models, and consider new applications and future developments.
\end{abstract}

First EPS Conference on Plasma Diagnostics - 1st ECPD,

14-17 April 2015

Villa Mondragone, Frascati (Rome) Italy

\footnotetext{
* Speaker.
} 